\title{
ELM flushing and Impurity Transport in the H-mode Edge Barrier in ASDEX Upgrade
}

\author{
T. Pütterich, R. Dux, M.A. Janzer, R.M. McDermott, \\ ASDEX Upgrade Team \\ Max-Planck-Institut für Plasmaphysik, EURATOM Association, D-85748 Garching, \\ Germany
}

\begin{abstract}
Density profiles of $\mathrm{He}^{2+}, \mathrm{C}^{6+}, \mathrm{Ne}^{10+}$ and $\mathrm{Ar}^{16+}$ are measured at ASDEX Upgrade with high temporal $(1.9 \mathrm{~ms})$ and radial resolution $(>3 \mathrm{~mm})$ using CXRS. This allows the transport coefficients at the edge transport barrier to be analyzed in between type-I ELMs during H-mode. All measurements resemble the neoclassical transport coefficients, which means that the diffusion coefficient ranges from $0.15-0.35 \mathrm{~m}^{2} / \mathrm{s}$ and the maximum of the inward convection velocity is Z-dependent increasing from $15 \mathrm{~m} / \mathrm{s}\left(\mathrm{He}^{2+}\right)$ to $90 \mathrm{~m} / \mathrm{s}\left(\mathrm{Ar}^{16+}\right)$. These results are combined into a simplified transport-erosion model for $\mathrm{W}$ which calculates the neoclassical transport coefficients self-consistently while taking several impurity species into account. The model also calculates erosion fluxes at the wall, prompt redeposition of $\mathrm{W}$ and loss terms due to parallel flows in the scrape-off layer. While several free parameters exist in the the model, it demonstrates that a quantitative consistency between the measured erosion fluxes of $\mathrm{W}$ and the observed $\mathrm{W}$ concentration inside the plasma is obtainable.
\end{abstract}

Key words:

PACS: 52.40.Hf, 52.25.Vy, 52.25.Fi, 52.55.Rk, 52.55.Fa

PSI-19 keywords: Impurity Transport, Edge Pedestal, Tungsten, ELM, ASDEX-Upgrade JNM keywords: F0800, P0500, P0600, S1300, T1000 
Corresponding and presenting author: Thomas Pütterich, Boltzmannstr.2, D-85748

Garching, Germany.

E-mail address: Thomas.Puetterich@ipp.mpg.de

\section{Introduction}

In H-mode plasmas the turbulent transport of energy and particles is strongly suppressed in a thin radial region at the edge of the confined plasma. The width of this edge transport barrier (ETB) is rather small (for ASDEX Upgrade $<2 \mathrm{~cm}$ ) with large radial gradients for $T_{e}, T_{i}$ and $\mathrm{n}_{e}$. In ELMy H-modes, edge-localized modes (ELMs) occur which make the steep gradients of temperature and density collapse for a few 100 microseconds. These recover again in the following milliseconds until the next ELM occurs. The transport details of this barrier determine how well the plasma confines energy enhancing its performance and how well it confines impurities and $\mathrm{He}$ ash which, in general, reduce the performance of a fusion plasma. The small spatial extent of the barrier and the fast change due to ELMs requires a diagnostic with good spatial and temporal resolution. In this work special focus is put on tungsten (W) as a plasma impurity. However, as a direct determination of the $\mathrm{W}$-transport profile was not possible, a Z-dependent measurement has been performed, which is used to predict the $\mathrm{W}$-transport by extrapolation. The edge charge exchange system at ASDEX Upgrade [1] was utilized for this, as it has a spatial resolution of up to $3 \mathrm{~mm}$ and is able to operate with a temporal resolution of $1.9 \mathrm{~ms}$ enabling the actual pedestal investigations.

The ETB is only one player controlling the impurity content of the plasma. Other influences are the time-dependent impurity sources at the first wall or the transport across and within the scrape-off layer (SOL). Both is influenced by the ELMs ejecting energy and particles from the plasma while the tens of milliseconds long phase between the ELMs does exhibit a considerably smaller erosion at the first wall and densities/temperatures in the SOL. The ultimate goal is to predict the impurity content of the confined plasma and thus an integral part is to quantitatively connect impurity erosion fluxes to the pedestal-top densities. A model is presented, which delivers this connection while including many experimentally observed details. It should 
be noted that the model is relatively simple and contains free parameters, which are chosen within a credible range to fit the measurements.

\section{Impurity Transport at the ETB}

\subsection{Determination of Transport Coefficients}

The impurity transport for $\mathrm{He}, \mathrm{C}, \mathrm{Ne}$ and $\mathrm{Ar}$ is measured by comparing the measured density profiles of specific ions from these impurities to a variety of results from a transport model, which are obtained by varying the diffusion coefficient and convection velocity at the ETB. In the following the details of the measurements are described and then the transport model is explained.

The edge CXRS system at ASDEX Upgrade covers the plasma edge at the outboard midplane with up to 8 different lines of sight (LOS) that are radially spaced by approximately $0.9 \mathrm{~cm}$. In order to fully resolve the steep gradients in impurity densities the plasma is swept by $2 \mathrm{~cm}$ in a $300 \mathrm{~ms}$ long phase during flattop. Along with the local CX-brightness the local ion temperature $\left(T_{i}\right)$ and toroidal rotation can be derived from the Doppler broadening and shift of the corresponding spectral line. The impurity density is calculated from the brightness of the spectral line using the local beam density of the $60 \mathrm{keV}$ heating beam and the reaction rate data from ADAS [2]. The plasma sweep allows also for good radial coverage by the Thomson scattering system, which measures the electron temperature $\left(T_{e}\right)$ and density profiles $\left(n_{e}\right)$. The electron density is measured additionally by utilizing the beam emission of a neutral Lithium beam penetrating the plasma edge. Inside the pedestal-top electron cyclotron emission is used to determine the core $T_{e}$. Interferometer measurements, which give line integrated densities, are used to restrict the electron density profiles at the pedestal-top. The uncertainty of the separatrix position from magnetic reconstruction can be minimized with the help of the steep $T_{e}$-profiles by setting $\mathrm{T}_{e, s e p}=85 \mathrm{eV}$ (typically $80-120 \mathrm{eV}$ at ASDEX Upgrade), a mandatory condition set by the parallel heat transport and power balance [3]. The $\mathrm{n}_{e}$-profiles from the Li-beam are then aligned to the measurements of the Thomson scattering. The CX-data are then aligned such that the steep $T_{i}$-gradient region coincides with that of the $T_{i}$-measurement (cf. figure 1). This 
process can be done within an accuracy of 2-3 $\mathrm{mm}$.

As a first step, the transport model is applied to the measurements of the $\mathrm{C}^{6+}$ density. In that case the data quality allowed for taking the evolution of several profiles within an ELM-cycle into account. In figure 1, the edge $T_{i^{-}}, T_{e^{-}}$and $n_{e}$-profiles are presented for two time points relative to an ELM in discharge \#22273. The parameters of the discharge are $\mathrm{I}_{p}=1 \mathrm{MA}, \mathrm{B}_{t}=-2.5 \mathrm{~T}$, $\mathrm{q}_{95}=4.5, \mathrm{P}_{a u x}=2.5 \mathrm{MW}, n_{e} / \mathrm{n}_{G W}=0.65$ and the ELM frequency is about $60 \mathrm{~Hz}$. The spacing between $\rho_{\text {pol }}=0.98$ and 1.00 corresponds to about $1 \mathrm{~cm}$ radial distance at the outer midplane of ASDEX Upgrade.

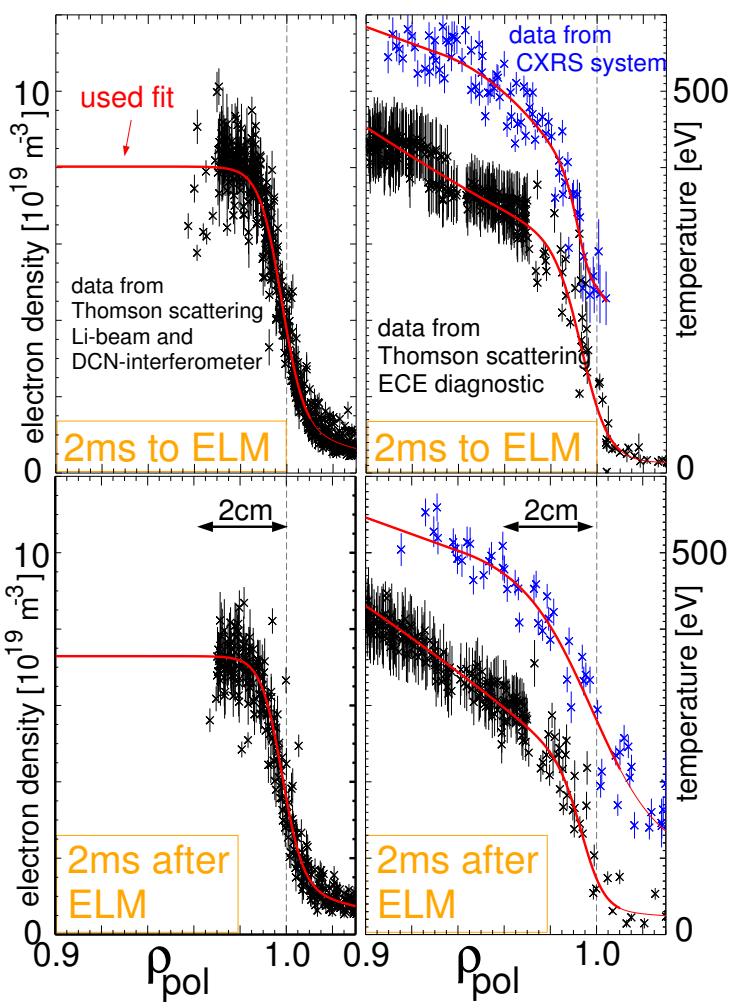

Fig. 1. Temperature and density profiles before and after an ELM as measured during the analyzed discharge phase of \#22273.

The evolution of the $\mathrm{C}^{6+}$ density profiles (cf. figure 2) was modeled with the impurity transport code STRAHL [4,5], which takes into account ionization and recombination rates [2] and calculates time-dependently the densities of all ionization stages along the plasma radius in a flux surface averaged manner. The local ionization and recombination rates are evaluated by taking the measured, time-dependent, electron profiles into account. The impurity transport is treated using D and v profiles. The model simulates ELM cycles until the C-density arrives at 
a quasi-equilibrium just modulated by the ELMs, while a constant carbon (neutral C) influx is assumed. In the model, the effects of an ELM are imitated by increasing D $\left(10 \mathrm{~m}^{2} / \mathrm{s}\right)$ for $200 \mu \mathrm{s}$ in the pedestal region, while the transport in-between ELMs is the subject of this investigation. For this the transport coefficients are assumed to be constant during the inter-ELM phase. This assumption is not far-fetched as the neoclassical transport coefficients do not vary strongly during the inter-ELM phase as will be shown later. It is known from earlier reports (e.g. [6]) that just inside the H-mode ETB there exists a region with relatively high turbulent (diffusive) transport (of the order of $1 \mathrm{~m}^{2} / \mathrm{s}$ ). For discharge \#22273, a slightly hollow $\mathrm{C}^{6+}$ density profile is found inside $\rho_{p o l}=0.96$. The model is adjusted for this by assuming a moderate outward pinch of $1.0 \mathrm{~m} / \mathrm{s}$ in this inner region. As the model aims to find the transport coefficients in the ETB

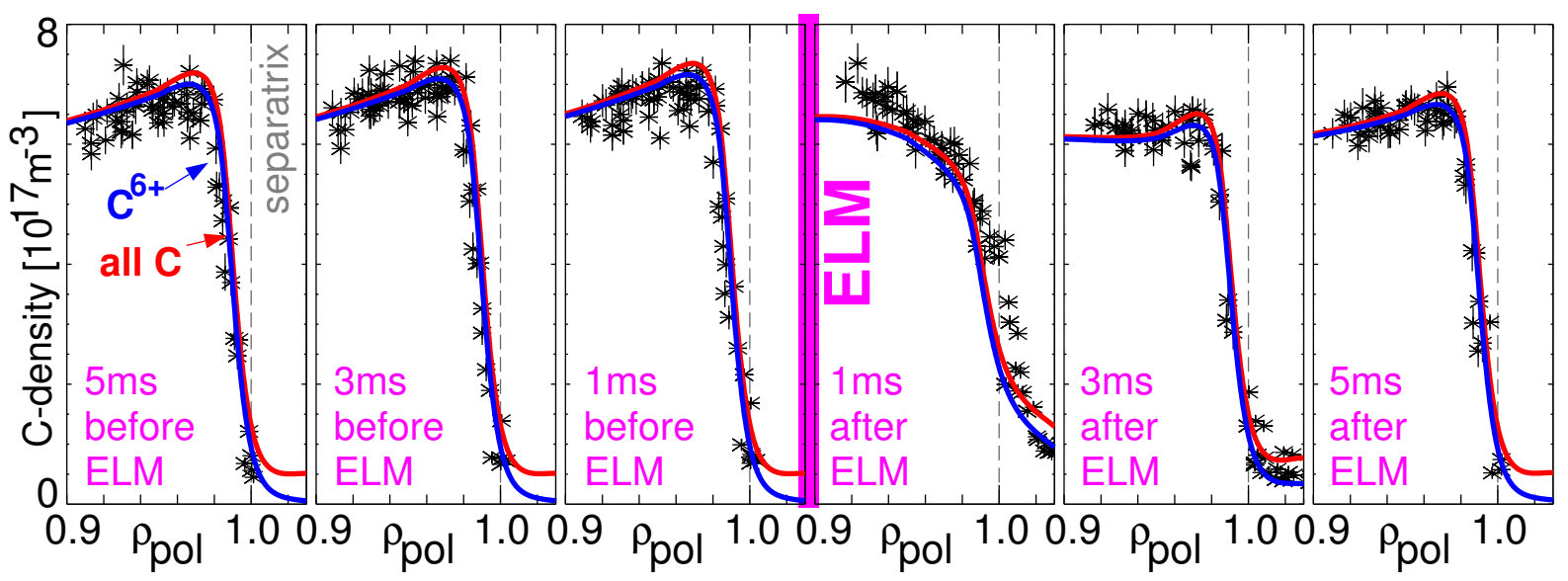

Fig. 2. Result of an ELM-synchronized data analysis from \#22273. A measured time sequence of the $\mathrm{C}^{6+}$ density (symbols) around a single ELM is presented along with the transport model results for the $\mathrm{C}^{6+}$ density (blue lines) and the total $\mathrm{C}$ density (red lines).

itself these adjustments do not matter.

In figure 2, the results for the best fit of $\mathrm{v}$ and $\mathrm{D}$ are presented. The agreement between data and model is quite good except for the phase $1 \mathrm{~ms}$ after the ELM. This time includes light from the dynamic ELM effects such as filaments. These effects are not included in the impurity model and therefore, the discrepancies are not surprising. It is astonishing that the steep gradients at the ETB are nearly fully restored within about $5 \mathrm{~ms}$ after the ELM crash. These steep gradients in the $\mathrm{C}^{6+}$ density which persist until the occurrence of the ELM, can only be modeled by including an inward drift. Local ratios of v/D at $\rho_{p o l}=0.99$ are found to be $-90 \pm 30 \mathrm{~m}^{-1}$ nearly independent of all other model assumptions. For the radial location of these steep gradients the 
absolute values of $\mathrm{v}$ matter and, thus, can be derived. This is done by a least square fit for which the algorithm adjusts $\mathrm{D}$ and $\mathrm{v}$ in the barrier region, while the value of $\mathrm{D}$ is assumed to drop in a small radial region to a constant value and the v-profile is represented by a Gaussian curve centered at the separatrix. The value of D and the maximum of the Gaussian used to describe $\mathrm{v}$ are subject to the fit. In order to determine $\mathrm{v}$ and $\mathrm{D}$ only the relative shape of the $\mathrm{C}^{6+}$ density profile between $\rho_{\text {pol }}=0.96$ and $\rho_{\text {pol }}=1.01$ and its evolution was considered. The best agreement (s. model curves in figure 2) is found for the transport coefficients depicted in figure 3 (black, solid). Evaluating the uncertainties of the fit-parameters, the diffusion coefficient is found to be in the range $0.1-0.6 \mathrm{~m}^{2} / \mathrm{s}$ at $\rho_{\text {pol }}=0.99$, where $\mathrm{v}$ is between $7-40 \mathrm{~m} / \mathrm{s}$. The transport coefficients in the ETB (compared at $\rho_{\text {pol }}=0.99$ ) are in agreement with neoclassical predictions for impurity transport $\left(\mathrm{D}_{\text {neo }}\right.$ and $\left.\mathrm{v}_{\text {neo }}\right)$ taken from the code NEOART [5,7]. NEOART calculates the collisional transport coefficients for an arbitrary number of impurities including collisions between them. The code solves the set of linear coupled equations for the parallel velocities in arbitrary toroidally symmetric geometry for all collision regimes. The classical fluxes are given by Eqs.(5.9) and (5.10) in [8]. The equations for the banana-plateau contribution are that in [9]. The Pfirsch-Schlüter contribution is calculated from the coupled equations (6.1-2) and (6.1415 ) in [8], as described in [10]. It may be noted, that the neoclassical prediction for D exhibits different shape as a function of $\rho_{\text {pol }}$ than the used model function, but the latter is supposed to take also the turbulent transport occurring at $\rho_{\text {pol }}<0.97$ into account.

For revealing the Z-dependence of D and $\mathrm{v}$ in the ETB, a discharge very similar to \#22273 was performed repeatedly and in each a different impurity density profile (intrinsic $\mathrm{He}^{2+}$ and $\mathrm{C}^{6+}$, puffed $\mathrm{Ne}^{10+}$ and $\mathrm{Ar}^{16+}$ ) was measured. The puff of $\mathrm{Ne}$ and $\mathrm{Ar}$ was designed such that for a phase of $600 \mathrm{~ms}$ an approximately constant pedestal-top density of the impurity was achieved. Due to the scatter in the data for Ne and Ar, the inter-ELM edge density profiles could not be analyzed time-dependently as was done for $\mathrm{C}^{6+}$ in discharge \#22273. However, it was found for the carbon density profile in \#22273 that it is nearly constant throughout a large fraction of the ELM cycle. Therefore, the measured data points from the inter-ELM phase $(5 \mathrm{~ms}$ after the ELM to 2 ms before the ELM) are accumulated and taken into account for the least square fit. Still, a separation of $\mathrm{v}$ and $\mathrm{D}$ is possible as the radius at which a specific ionization stage exists depends on the absolute value of v. In figure 4, these measurements are presented along with the model curves which result from the inter ELM transport profiles as shown at the bottom of 


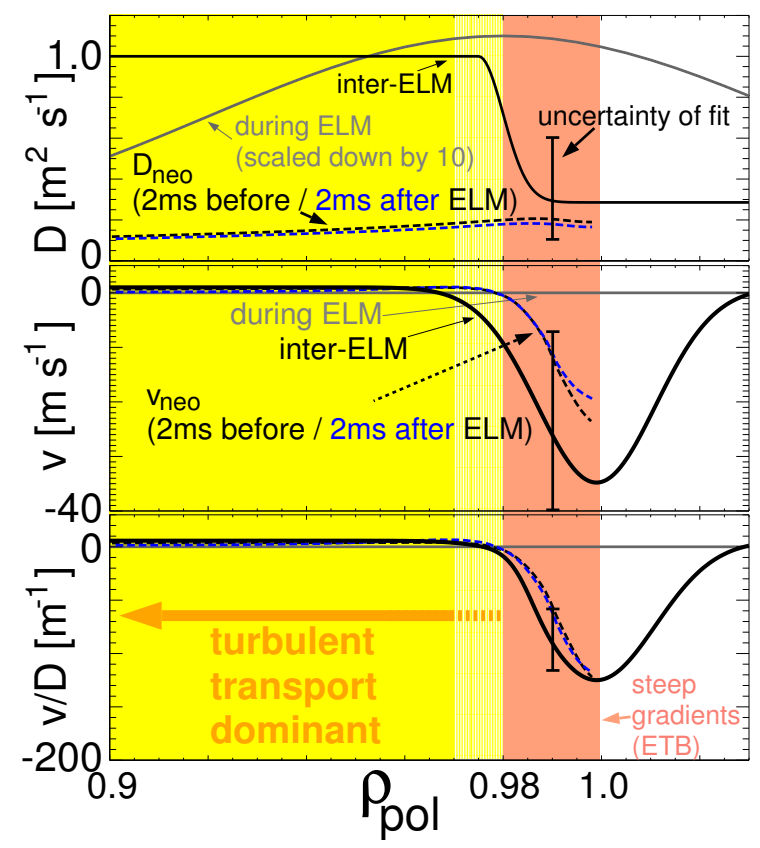

Fig. 3. The transport coefficients for $\mathrm{C}$, as obtained by the presented analysis, are compared to neoclassical transport coefficients. Due to the additional turbulent transport inside of $\rho_{p o l}=0.97$ the comparison is only relevant in the ETB region.

the figure. There, the neoclassical prediction is also depicted. The two presented neoclassical profiles represent the results for the two background profiles just after and just before the ELM as presented in figure 1. Included in the prediction is also the influence of the concentrations of $\mathrm{He}, \mathrm{C}$ and $\mathrm{O}$ as measured by CXRS or survey spectrometers. The neoclassical curves deliver good agreement in the pedestal region, while the uncertainties in the individual $\mathrm{v}$ and $\mathrm{D}$ values (colored bars just inside separatrix) are considerable. The uncertainties in the v/D values are smaller. For higher charged impurities a stronger inward pinch is necessary to explain the observed impurity density data, which is exactly what neoclassical theory predicts. The fit results for v/D evaluated at $\rho_{\text {pol }}=0.99$ are presented in figure 5, along with the pedestal peaking factor $F_{I}$. The latter contains the integrated effects of pedestal transport, i.e. here between separatrix and $\rho_{p o l}=0.97$, and it is defined as $F_{I}=\frac{n_{I}\left(\rho_{p o l}=0.97\right)}{n_{I}\left(\rho_{p o l}=1.0\right)}=\exp \left[\int_{\rho_{p o l}=1.0}^{\rho_{p o l}=0.97} \frac{v}{D} d r\right]$, where $n_{I}$ is the impurity density. This expression is accurate only for a real equilibrium without a source term. Nevertheless, it shall be used here as a figure of merit. For comparison, the neoclassical values for v/D and the pedestal peaking factor $F_{I}$ are also presented and within the uncertainties agreement is found. 


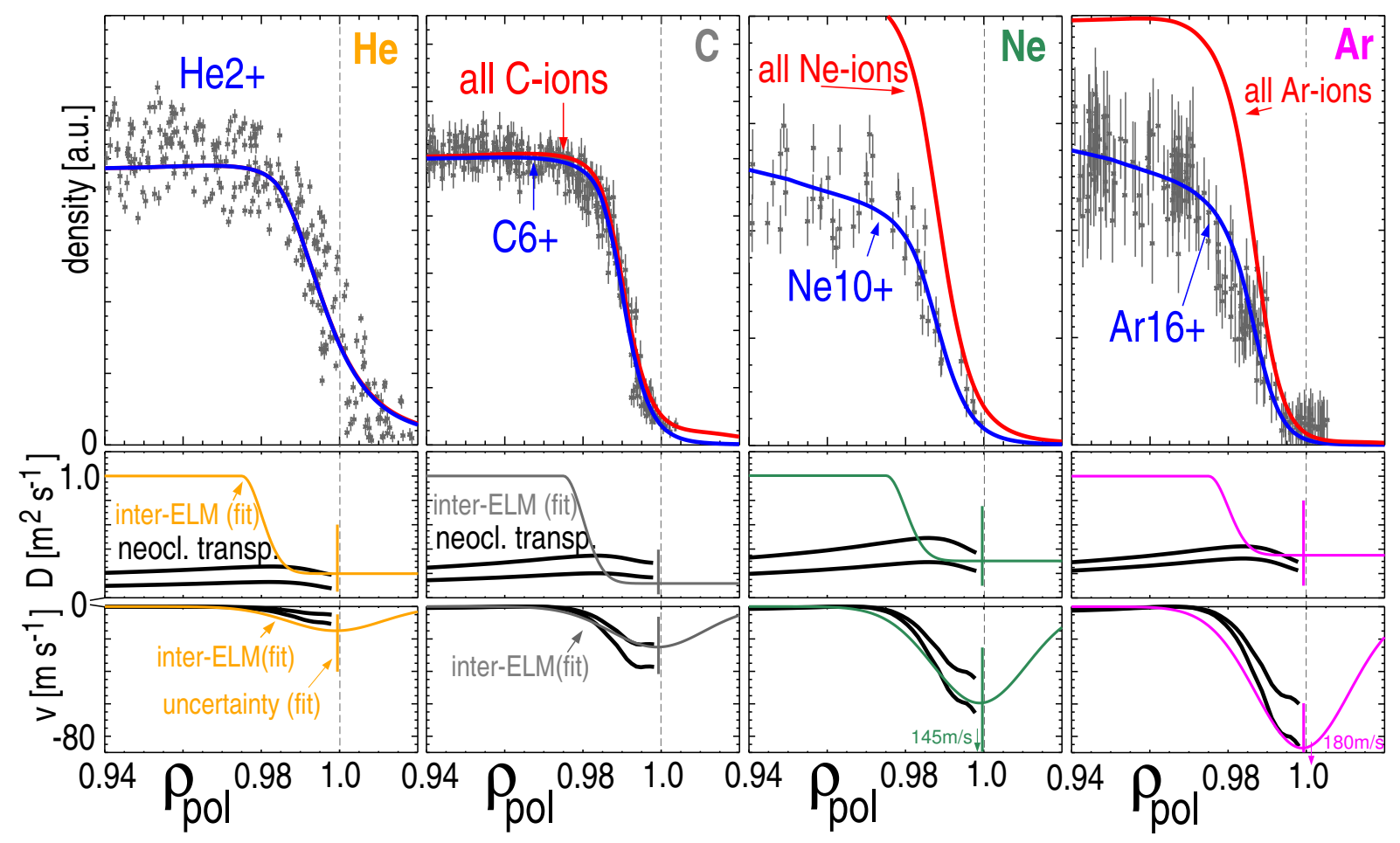

Fig. 4. top: Impurity ion densities (symbols), i.e. for $\mathrm{He}^{2+}, \mathrm{C}^{6+}, \mathrm{Ne}^{10+}$ and $\mathrm{Ar}^{16+}$, as measured during the inter-ELM phase are presented along with the best fit of modeled profiles for the specific ions (blue) and the total impurity densities (red); bottom: The underlying transport coefficients are presented and compared to the neoclassical coefficients.
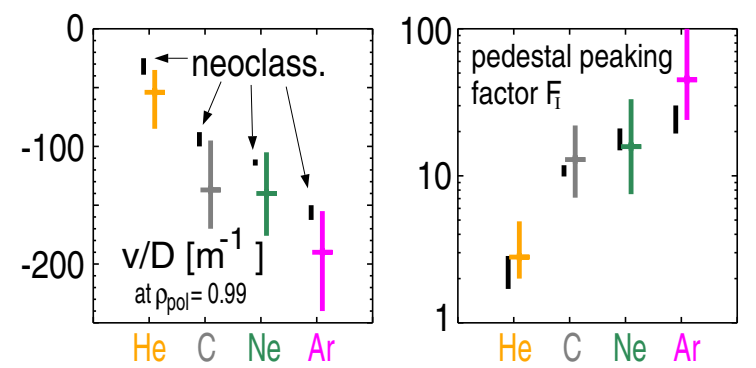

Fig. 5. The resulting ratios v/D and the pedestal peaking factors $F_{I}$ (orange, gray, green and magenta) are compared to neoclassical values (black).

\subsection{Discussion}

The results indicate that turbulent transport is of negligible importance for the impurities at the edge transport barrier and thus, transport arrives at the neoclassical level. This is in agreement with the results of earlier investigations from ASDEX Upgrade [6], ASDEX [11], C-Mod [12], DIII-D [13] and JET [14], all of which lacked the charge stage resolved information and were performed for only single impurities. A clear sign of neoclassical transport is also that the 
inward pinch follows the neoclassical scaling as it is stronger for higher charged impurities. This leads to the conclusion that each impurity has a different gradient at the ETB. The transport parameters depend slightly on the local impurity mix, as the neoclassical transport for one impurity species is the result of its friction with the background ions and the various other impurities. For W, which exhibits charge states of about 15-25 at the ETB, the largest ratios of v/D apply. This means that for a given pedestal in $T_{e}, T_{i}$ and $n_{e}$ the impurity transport can be predicted. However, the physics that determines the background profiles is not explained by the recent measurements.

It should be noted that neoclassical theory breaks down for deuterium at the pedestal, but stays valid for the impurity species. There are two reasons for this: On the one hand the banana widths are smaller for impurities and additionally, the banana and plateau contributions for the impurity transport are negligible at the radii in question. Thus, only Pfirsch-Schlüter (PS) Transport is of importance.

\section{Connecting ETB-Transport and Wall-Erosion}

The measurements described in section 2 call for an integrated modeling approach, in which several impurities are taken into account to predict the transport of W. In this section a simplified model is described, which allows for connecting the pedestal-top W density to the erosion fluxes at the first wall. Such a model involves free parameters that can be adjusted within credible ranges to obtain a match between the model results and the measurements. In order to keep the number of free parameters small, the complicated processes at the plasma edge were targeted with a $1.5 \mathrm{D}$ impurity transport code. Simplifications were introduced not only to reduce the 3D geometry but also to handle complex systems like the SOL with only a few parameters. A more complex model would need to be filled with more assumptions, i.e. free parameters. Thus, the model is an attempt to demonstrate how the various processes might combine. In fact, the model gives a possible explanation for a peculiar feature observed for the $\mathrm{W}$ confinement time, which will be described below.

In the actual model $\mathrm{C}, \mathrm{O}$ and $\mathrm{W}$ are modeled simultaneously and the transport coefficients for each species are determined dynamically in the ETB, depending on the local impurity densities. 
For W, also the erosion flux from the first wall is dependent on the impurity mix of the plasma which contacts the first wall as $\mathrm{W}$ erosion is almost exclusively done by light impurities. When the ELM brings impurities to the wall, the pedestal-top densities of the impurities influence the wall sputtering. This gives the gross erosion of $\mathrm{W}$, which can be compared to the spectroscopic measurement. Part of the eroded material is promptly redeposited and the rest enters the plasma in the SOL. Here, the radial transport competes with the parallel losses. When the impurity ions enter the confined plasma, the measured transport coefficients apply closing the loop.

\subsection{Erosion and Prompt Re-deposition}

The erosion fluxes of $\mathrm{W}$ are measured in the experiment at numerous locations and with high time-resolution [15] and a variation of the erosion is observed within an ELM cycle as the ELM is responsible for a major part of the erosion [15]. It was demonstrated before [15] that the outboard limiters are by far the most important W source for the confined plasma. In the model this is reflected by the choice that only $1 / 3$ of the total low-Z impurity losses were considered for the $\mathrm{W}$ production at the outboard limiters. In the model, the transport coefficients, the impurity densities and the temperatures at the first wall define the gross erosion of $\mathrm{W}$. The sputtering yield [16] for normal incidence, which is a function of impurity mass and energy, is used. The energy at the surface is approximated by $E=k_{B}\left(2 T_{i}+3 Z T_{e}\right)$, where the second term describes the acceleration in the sheath, which depends on the ion charge. The ionization stage distribution varies during an ELM cycle, when higher charged ions are swept out from the ETB and hit the limiter. An example of the temporal evolution of the $\mathrm{W}$ influx is given in the upper left graph

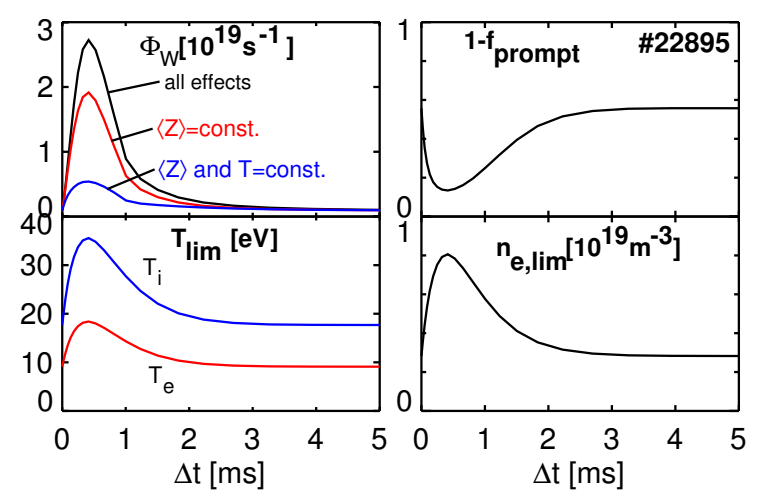

Fig. 6. Temporal evolution of the tungsten influx and the fraction of not promptly redeposited W during an ELM cycle. The used values for $T_{e}, T_{i}$ and $n_{e}$ at the limiters are shown in the lower graphs. 
of figure 6, which gives results from the modeling of a phase with $100 \mathrm{~Hz}$ ELM frequency in discharge \#22895. The blue curve shows the influx change due to the increased losses of $\mathrm{C}, \mathrm{O}$, and $\mathrm{W}$ without considering the changes in the sputtering yield. For the red curve, only the temperature change at constant charge distribution is considered, while the black curve is calculated taking all effects into account. The sputtering is very sensitive to the temperatures at the limiters. As these temperatures are not known to the necessary accuracy, the temperatures were adjusted (using $T_{i}=2 T_{e}$ ) such that the measured $\mathrm{W}$ influx during the inter-ELM phase and during the ELM was reproduced. Thus, the erosion model can't give much insight for itself, it just allows to close the loop between the confinement of light impurities and the $\mathrm{W}$ erosion.

From the energy of the incident impurity a mean neutral velocity for the sputtered $\mathrm{W}$ is calculated assuming a Thompson [17] distribution. The ionization length of $\mathrm{W}$ is of the order of the Larmor radius of the ionized $\mathrm{W}$ and a fraction $f_{\text {prompt }}$ of ionized $\mathrm{W}$ returns to the surface on the first travel along the Larmor radius. This prompt redeposition effect is calculated for the sputtering by each ion species using a $\cos \theta$ angular velocity distribution of the sputtered $\mathrm{W}$. In figure 6, the upper right graph shows the part of the ionized $\mathrm{W}$ that is not promptly redeposited, where the toroidal field was $B_{t}=2.5 T$. During the ELM, the rise of $n_{e}$ and $T_{e}$ leads to shorter ionization lengths and thus enhanced prompt redeposition.

\subsection{Parallel Losses in the SOL}

In the SOL, the parallel transport towards the divertor or to the limiting elements in the main chamber is described by volumetric losses with a loss frequency that is given by the parallel connection length between the surface elements $\left(L_{\|}\right)$and the mean flow velocity of the deuterium ions: $\nu_{\|}=\left(2 M / L_{\|}\right) \sqrt{k_{B}\left(3 T_{i}+T_{e}\right) / m_{D}}$, where the Mach number $M$ was set to 0.1 for the presented results. At a certain radius in the SOL, the main chamber limiters are introduced into the code by switching the connection length from $50 \mathrm{~m}$ to $1 \mathrm{~m}$. The neutral impurities start with a prescribed influx rate at this limiter position. They have a constant radial velocity $v_{0}$ and their density decays radially according to the rate $\nu_{i o n}=n_{e}\left\langle\sigma_{i o n} v_{e}\right\rangle$ for ionization: $n_{0}(r)=n_{0}\left(r_{\text {lim }}\right)\left(r_{\text {lim }} / r\right) \exp \left[-\int\left(\nu_{\text {ion }} / v_{0}\right) d r\right]$. 


\subsection{Radial Transport of Impurities}

For the radial transport, which is mainly collisional, the light impurities and $\mathrm{W}$ are included in the calculation from the SOL, across the ETB up to the pedestal-top. As has been described above, neoclassical transport is found to be applicable at the ETB. The calculation of the transport is also extended to the SOL, while only the PS transport is taken into account as the contributions from the banana and plateau regime are negligible. The diffusion coefficient due to collisions increases with increasing low-Z impurity concentration. Furthermore, the low-Z impurities lead to a dilution of the deuterium ions. Thus, the $n_{D}$ profile has a decreasing gradient with increasing low-Z concentration in the ETB and the PS inward pinch of $\mathrm{W}$ decreases. The

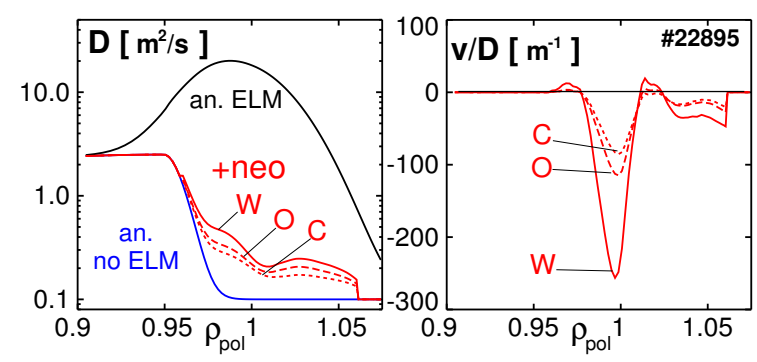

Fig. 7. Profiles of diffusion coefficient and drift parameter for the modeling of discharge \#22895.

resulting transport coefficients of the radial transport model are depicted in figure 7, which is again for discharge \#22895. In the phase between ELMs, the anomalous diffusion coefficient (blue curve) was reduced in the ETB to a very low value of $0.1 \mathrm{~m}^{2} / \mathrm{s}$. The collisional values from classical and PS transport are larger and exhibit the highest values for $\mathrm{W}$. The total value of the drift parameter v/D in this phase are shown on the right, where $\mathrm{W}$ has the strongest inward drift as it exhibits the highest charge at the ETB. The mean charge at poloidal flux label $\rho_{\text {pol }}=0.99$ is 5.6 for $\mathrm{C}, 7.4$ for $\mathrm{O}$ and 16 for W. An ELM is induced by a sudden switch-on of a large diffusion coefficient in the edge (black curve), which decays linearly within $1 \mathrm{~ms}$.

\subsection{Model Results and Discussion}

The resulting evolution of the total W density during an ELM cycle of discharge \#22895 is shown in figure 8 . The cycle has been repeated up to quasi-equilibrium, i.e. the density profile at the end and at the beginning of the ELM cycle are equal. The W density gradient around 


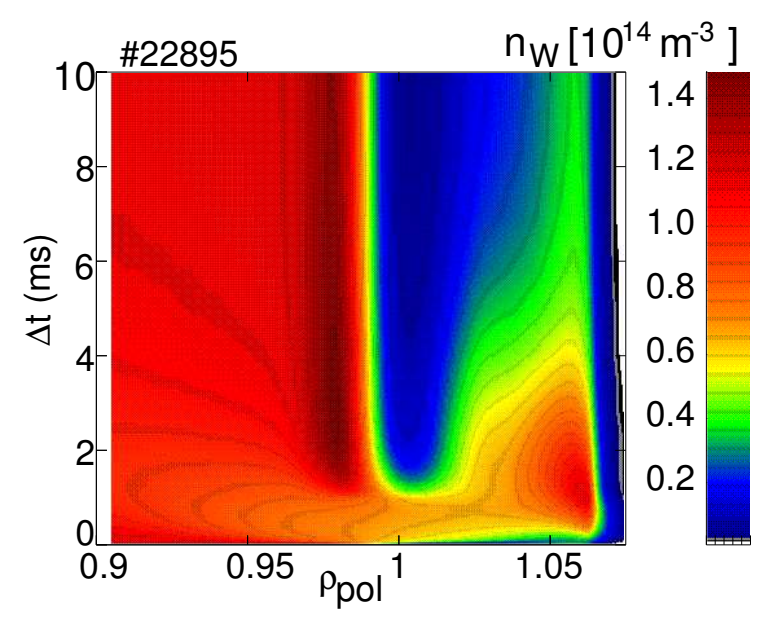

Fig. 8. Modeled evolution of the total W density during one ELM cycle.

the separatrix is strongly degraded during the ELM and the increasing source from the limiter, which is at $\rho_{\text {pol }}=1.063$, leads to a density rise in the outer SOL. After the ELM, the source decreases and a strong density gradient forms in the ETB. The ratio of the $\mathrm{W}$ density at $\rho_{\text {pol }}=0.9$ to the value at $\rho_{p o l}=1$ is 22 at the end of the cycle and 7.2, when taking the temporal average during an ELM cycle. For a similar discharge \#22901, which had 10 times less deuterium fueling and an ELM frequency of about $50 \mathrm{~Hz}$, the respective density ratios were 51 and 17.

Three discharge phases with different deuterium puff levels $\Phi_{D}$ and ELM frequencies were modeled \#22898: $\Phi_{D}=1.5 \cdot 10^{22} \mathrm{~s}^{-1}, f_{E L M} \approx 200 \mathrm{~Hz}, \# 22895: \Phi_{D}=10^{22} \mathrm{~s}^{-1}, f_{E L M} \approx 100 \mathrm{~Hz}$, and \#22901: $\Phi_{D}=10^{21} \mathrm{~s}^{-1}, f_{E L M} \approx 50 \mathrm{~Hz}$. Time-dependent profiles of $T_{e}, T_{i}$ and $n_{e}$ were fitted for equal time delays relative to the ELM start using the data from a $500 \mathrm{~ms}$ long period.

The carbon influx level was chosen to match the measured C concentration at $\rho_{\text {pol }}=0.95$ which was $0.9 \%$ for \#22898, $1.2 \%$ for \#22895, and $2.1 \%$ for \#22901. The oxygen influx was set to $1 / 4$ of the carbon influx.

The plasma temperatures in front of the limiters were adjusted to match the $\mathrm{W}$ erosion flux and the used inter-ELM and ELM values for $T_{e}$ at the limiter were $8.4 \mathrm{eV} / 12 \mathrm{eV}$ for \#22898, $10 \mathrm{eV} / 20 \mathrm{eV}$ for \#22895, and $12 \mathrm{eV} / 35 \mathrm{eV}$ for \#22901. All of these values are consistent with typical temperatures from measurements (e.g. [18]).

The transport model was solved for three artificial scans of the ELM frequency with $f_{E L M}=$ $0,50,100$, and $200 \mathrm{~Hz}$ using one of the three settings at a time for the background profiles, limiter temperatures and low-Z fluxes. Figure 9 shows a number of key quantities for these 

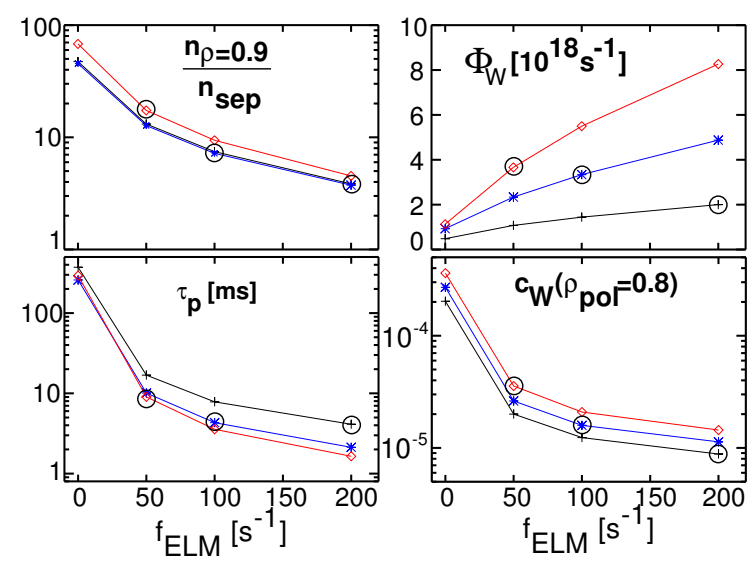

Fig. 9. Calculated edge density ratio, source rate, particle confinement time and $\mathrm{W}$ concentration for a scan of the ELM frequency. Different colors correspond to the use of the edge $T_{e^{-}}, T_{i}$ and $n_{e}$-profiles from the discharges \#22898 (black, $200 \mathrm{~Hz}$, coldest at limiter), \#22895 (blue, $100 \mathrm{~Hz}$ ) and \#22901 (red, $50 \mathrm{~Hz}$, hottest at limiter). Circles correspond to the experimental values for the three discharges. All quantities are temporal averages of one ELM cycle.

scans. Black curves are for the settings of \#22898 (200 Hz, coldest at limiter), blue for \#22895 $(100 \mathrm{~Hz})$, and red for \#22901 (50 Hz, hottest at limiter). The black circles surround the points for which the limiter temperatures, ELM sizes, and influx values were adjusted to the measurement. The upper left box shows the strong decrease of the $\mathrm{W}$ density peaking with increasing ELM frequency, which can reach values on the order of 100 for the artificial zero ELM case. The plasma with the lowest puff level has the highest peaking factors. The particle confinement time $\tau_{p}$ of $\mathrm{W}$ (lower left box) is calculated with the total number of $\mathrm{W}$ ions in the confined region $N_{W}$ and the total source $\Phi_{W}$. It decreases strongly with the ELM frequency. A hotter SOL leads to higher losses in the SOL which explains that $\tau_{p}$ for the medium sized gas puff is below the value of the highest puff case. In the upper right box, the increase of the erosion source with $f_{E L M}$ is due to the linear picture without taking the change of the ELM size and the lower inter-ELM temperatures into account. The encircled points reflect the measured behavior. Nevertheless, the rise of the source is not strong enough to beat the change in $\tau_{p}$ and the $\mathrm{W}$ concentration $c_{W}$ (relative to $n_{e}$ ) at $\rho_{p o l}=0.8$ decreases on each of the linear curves, as shown in the lower right box. Even though the model reproduces the experimental values of $c_{W}$ within $10 \%$, we consider the model only to be a consistent description based on many ad hoc assumptions, which need further detailed experimental investigations.

It should be noted that free parameters exist in the model, which were chosen within credible 
ranges to obtain a good match between measurements and model. These parameters are:

- the SOL and limiter temperatures, which influence parallel losses in the SOL and erosion at the limiter

- the parallel Mach number, which influences parallel losses in the SOL

- the level of turbulent, radial transport in the SOL

- the length and impact of an ELM

Furthermore, following simplifications have been assumed:

- the 3D structure of the SOL is replaced by simple volume losses with characteristic loss times

- the radial transport is modeled with diffusion and convection

- the ELM is modeled as an increased radial diffusion for impurities at the plasma edge and SOL

- the erosion model is idealized and simplified, resulting in a Thompson distribution for the eroded $\mathrm{W}$ atoms

- the prompt re-deposition of $\mathrm{W}$ does not consider electric fields and surface roughness

However, the strength of the model is the connection between erosion and confined plasma, while leaving not an arbitrary amount of freedom for the free parameters: The impurities influence the radial transport coefficients and erosion. The SOL and limiter temperatures are connected via an exponential that connects to the measured separatrix temperatures. The limiter temperatures are given by the measured $\mathrm{W}$ erosion fluxes. The Mach number of 0.1 in the SOL is fixed for all modeled cases. The mechanisms described in the model offer also an explanation for the fact that with increasing ELM frequency the particle confinement first decreases and then increases again for even higher ELM frequencies.

\section{Summary and Outlook}

At ASDEX Upgrade, the 1D transport coefficients for impurities in the edge transport barrier (ETB) have been measured for $\mathrm{He}^{2+}, \mathrm{C}^{6+}, \mathrm{Ne}^{10+}$ and $\mathrm{Ar}^{16+}$ during the inter-ELM phase in type-I ELMy H-mode. This was accomplished by modeling the impurity densities as measured by the edge CXRS system with the impurity transport code STRAHL. The values of v/D at the 
ETB are restricted by the measurements with small uncertainties. Even the individual values of $\mathrm{v}$ and $\mathrm{D}$ can be determined, however, with larger uncertainties. The transport coefficients are found to be neoclassical in absolute value and the neoclassical Z-dependence, i.e. an increasing inward pinch for higher charged impurities, is observed.

Independently from these measurements, a model was developed which for $\mathrm{W}$ relates the ETB transport, the erosion fluxes at the wall and the transport across the scrape-off layer to each other. This approach is seen to be especially worthwhile for $\mathrm{W}$, as its transport at the ETB is dependent on the other impurities and its erosion at the wall is a result of impurity sputtering. The model incorporates some free parameters that were used to match the experimentally observed $\mathrm{W}$ concentrations within $10 \%$. The free parameters were chosen within credible ranges which suggests that the model captures the important physics of the processes involved. Still, the model can only be considered as a guideline for investigations until the inherent assumptions can be experimentally validated. It should be noted, that the pedestal in the density of the background deuterium ions is not explained or understood by the actual work, but it is the main drive of the described transport processes at the ETB.

\section{References}

[1] T. Pütterich et al., Physical Review Letters 102, 025001 (2009).

[2] H. P. Summers, The ADAS User Manual, version 2.6 http://adas.phys.strath.ac.uk (2004).

[3] J. Neuhauser et al., Plasma Physics and Controlled Fusion 44, 855 (2002).

[4] K. Behringer, 'Description of the impurity transport code STRAHL', JET-R(87)08, JET Joint Undertaking, Culham (1987).

[5] R. Dux, Technical Report No. 10/30, IPP, Garching, Germany.

[6] R. Dux, Fusion Science and Technology 44, 708 (2003).

[7] A. G. Peeters, provided the NEOART code, 1998.

[8] S. P. Hirshman and D. J. Sigmar, Nuclear Fusion 21, 1079 (1981).

[9] W. A. Houlberg, K. C. Shaing, S. P. Hirshman, and M. C. Zarnstorff, Physics of Plasmas 4, 3230 (1997). 
[10] A. G. Peeters, Physics of Plasmas 7, 268 (2000).

[11] ASDEX Team, Nuclear Fusion 29, 1959 (1989).

[12] T. S. Pedersen et al., Nuclear Fusion 40, 1795 (2000).

[13] M. E. Perry et al., Nuclear Fusion 31, 1859 (1991).

[14] D. Pasini et al., Plasma Physics and Controlled Fusion 34, 677 (1992).

[15] R. Dux et al., Journal of Nuclear Materials 390-391, 858 (2009).

[16] W. Eckstein, C. García-Rosales, J. Roth, and W. Ottenberger, Technical Report No. IPP 9/82, MaxPlanck Institut für Plasmaphysik, Garching, Germany.

[17] M. W. Thompson, Phil. Mag. 18, 377 (1968).

[18] M. Kočan et al., PSI 2010, San Diego, submitted to J. Nucl. Mater. (2010). 